\title{
Artesanado para la comunicación de la identidad cultural y patrimonio. Proyecto réplica de baldosas en la Universidad de Granada
}

Rosario Velasco Aranda1 / Joan Sanz Sánchez² Universidad de Granada

\section{Resumen:}

La artesanía como parte del diseño del plan estratégico para el relanzamiento y actualización de La Tienda Universidad de Granada. Esta iniciativa está vinculada a la investigación traducida en diseño de producto, como reflejo del contexto local y cultural que define tanto a esta universidad, a su historia, su geografía, patrimonio, autenticidad e idiosincrasia. Una forma única de hacer y entender, con carácter original, local y sostenible. Los productos comerciales generados por esta institución tienen una doble función, ser imagen y respirar universidad, así como reforzar los valores de la ciudad a la que representa, ya que tiene un posicionamiento nacional e internacional, por la afluencia turística que recibe la ciudad.

Palabras clave: artesanía, diseño, producto, tendencia, cultura y patrimonio.

Crafts in the "La Tienda de la Universidad de Granada".

Project replica hydraulic tiles chapel Colegio Máximo de Cartuja.

\begin{abstract}
Crafts as part of the objective in the design of the strategic plan for the relaunch and update of "La Tienda de la Universidad de Granada". This initiative is linked to research translated into product design, as a reflection of the local and cultural context that defines this university, its history, geography, heritage, authenticity and idiosyncrasy. A unique way of doing and understanding, with original, local and sustainable character. The commercial products generated by this institution have a double function, being an image and breathing university, as well as reinforcing the values of the city it represents, since it has a national and international positioning, due to the tourist influx that the city receives.of each project, aiming to generate a broad view of Sociotechnical Innovations.
\end{abstract}

Keywords: craft, design, product, trend, culture and heritage.

\footnotetext{
Rosario Velasco Aranda es doctora y profesora asociada en el departamento de Dibujo de la Facultad de Bellas Artes de Granada, también es ilustradora y diseñadora autónoma. Su carrera profesional se ha ido ampliando en profundidad, en el mundo del diseño, en sus distintas aplicaciones, trabajo en publicidad, estampación textil, prensa, diseño de producto e interiorismo. Colabora con varios estudios de diseño, agencias, editoriales... Aparte de su trayectoria profesional como diseñadora e ilustradora, dedica gran parte de su tiempo a la actividad investigadora como investigadora principal en el «Proyecto y diseño de La Tienda de la Universidad de Granada». Correo-e: rosariova@ugr.es

2 Joan Sanz Sánchez es licenciado en Bellas Artes por la Universidad de Granada, con estudios de doctorado en el máster oficial «Tipografía. Disciplina y usos» de la Universidad de Barcelona, y con estudios de posgrado en «Gestión de proyectos multimedia» en el Centro Universitario de Diseño y Arte de Barcelona Eina. Ilustrador y diseñador gráfico es colaborador habitual de diversas editoriales y publicaciones de ámbito nacional y desarrolla paralelamente su carrera docente como profesor en materias de diseño en la facultad de Bellas Artes de Granada y en Enseñanzas Artísticas Superiores. Realiza su tesis doctoral sobre «La comunicación gráfica en la imagen institucional universitaria» abordada desde la práctica del rediseño de la marca institucional y el desarrollo de su implementación en la Universidad de Granada.
} 


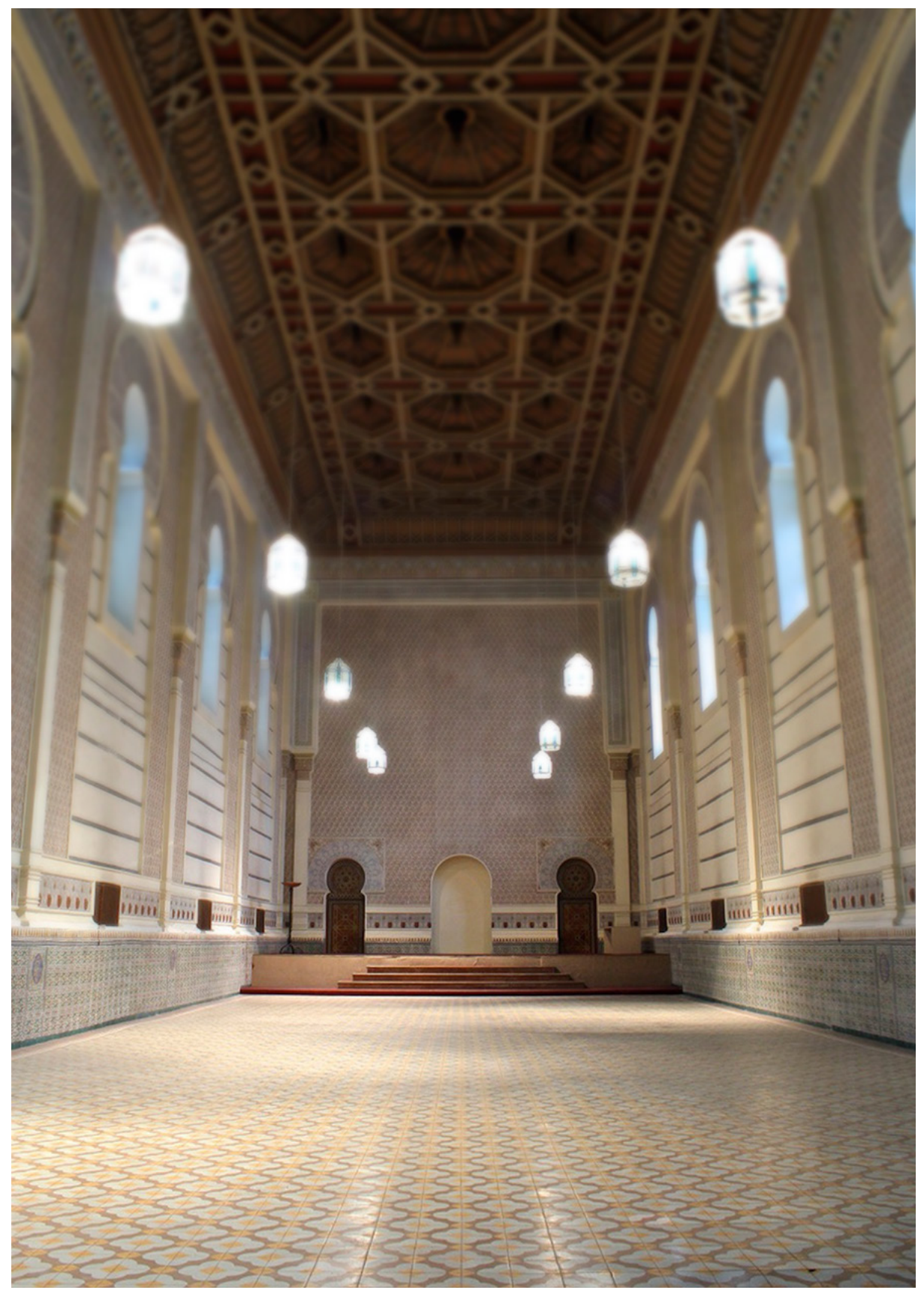

Fig. 1. Capilla neomudéjar Colegio Máximo de Cartuja. Fotografía Ángel García Roldán

El reciente resurgir de la artesanía se origina, entre otras cuestiones, gracias a programas que han contribuido a la mejora del acceso de los productos artesanos a nuevos canales de comercialización nacionales o internacionales. La Constitución Española, en su artículo 130.1, dispone que los poderes públicos atenderán a la modernización y desarrollo de todos los sectores económicos, entre los que se cita expresamente a la artesanía, con el fin de equiparar el nivel de vida de todos los españoles ${ }^{3}$. 
Por su lado, el Gobierno Andaluz, responsable y consecuente de la importancia y significado que tiene el artesanado producido en Andalucía, aprobó la Ley 15/2005, de 22 de diciembre, de Artesanía de Andalucía, con el fin de promocionar la actividad artesanal, así como reconocer a la artesanía su valor como fuente generadora de empleo, medio de cohesión social y recurso turístico de alta potencialidad, así como la divulgación de todas aquellas manifestaciones artesanales de interés tradicional o de arraigo en la Comunidad Autónoma de Andalucía, a fin de recuperar la importancia cultural, social y económica que le corresponde, mejorando de esta forma el acceso de los artesanos y artesanas al mercado 4 . Un paso significativo que ayuda a reconocer, valorar y rescatar procesos tradicionales con el fin de preservar la esencia de una identidad particular y única, así como fomentar su adaptación al mercado actual por medio de proyectos y programas que contribuyan a ese reconocimiento.

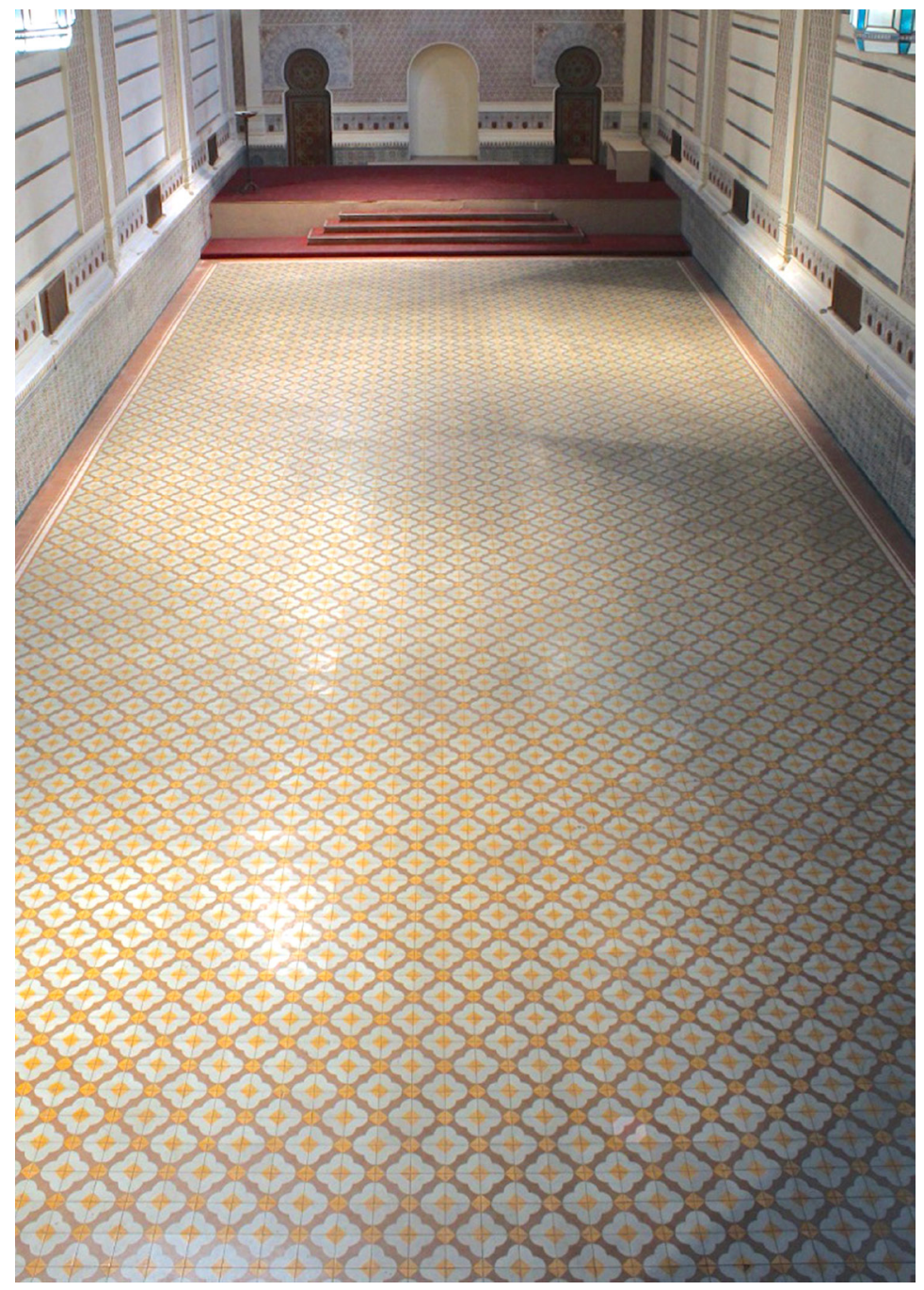

Fig. 2. Solería capilla neomudéjar Colegio Máximo de Cartuja. Fotografía Ángel García Roldán

4 Ley 15/2005, de 22 de diciembre, de Artesanía de Andalucía. https://www.boe.es/buscar/pdf/2006/BOE-A-2006-759-consolidado.pdf

Estatuto de Autonomía de La Junta de Andalucía.

http://www.juntadeandalucia.es/export/drupaljda/lo_2-2007.pdf 
Un ejemplo es el caso que desde el Vicerrectorado de Extensión Universitaria de la Universidad de Granada se impulsa a través del programa de investigación «Proyecto y diseño de La Tienda de la Universidad de Granada», vinculado a dicho Vicerrectorado y a la Oficina de Transferencia del Conocimiento (OTRI). Este proyecto tiene como objetivo el diseño del plan estratégico para el relanzamiento y actualización de La Tienda Universidad de Granada, el estudio y desarrollo de líneas de objetos acordes a los valores que la universidad debe mostrar en su espacio comercial a través de sus productos. Como consecuencia y para el desarrollo de este proyecto, se atiende a la necesaria colaboración, entre otros, de productores locales y artesanos. Con este programa se pretende que La Tienda Universidad de Granada, sea laboratorio creativo, germen facilitador de inquietudes y aptitudes creativas del artesanado andaluz. Así como servir a la visibilidad y posición de los productos artesanos en los canales comerciales físicos y online de la Universidad de Granada.

Como muestra de modernización e impulso de la artesanía elaborada en Andalucía ejemplificamos con el proyecto Baldosas Hidráulicas, labor y línea de desarrollo de producto en conjunción con el trabajo de profesionales, alumnos egresados y profesores de la Universidad de Granada. Este proyecto trata sobre la réplica de la solería de la capilla neomudéjar del Colegio Máximo de Cartuja, mediante el rescate de los procesos -ya casi olvidados - de la producción de baldosa hidráulica. Este proyecto fue diseñado y coordinado por la profesora de la Facultad de Bellas Artes de Granada y diseñadora Rosario Velasco y ejecutado por Mosaicos La Mar de Lejos. Este es un proyecto surgido y producido en Granada, donde su inspiración procede de la armonía entre arte, evocación granadina y su compromiso con el medio ambiente. Producto artesano, cada piezas está fabricada a mano de una en una. Tanto los materiales empleados cómo el proceso tiene con un alto compromiso medioambiental, los pigmentos son naturales y de gran calidad, y el proceso mantiene la técnica original de hace más de un siglo, obteniendo un producto $100 \%$ sostenible y local.

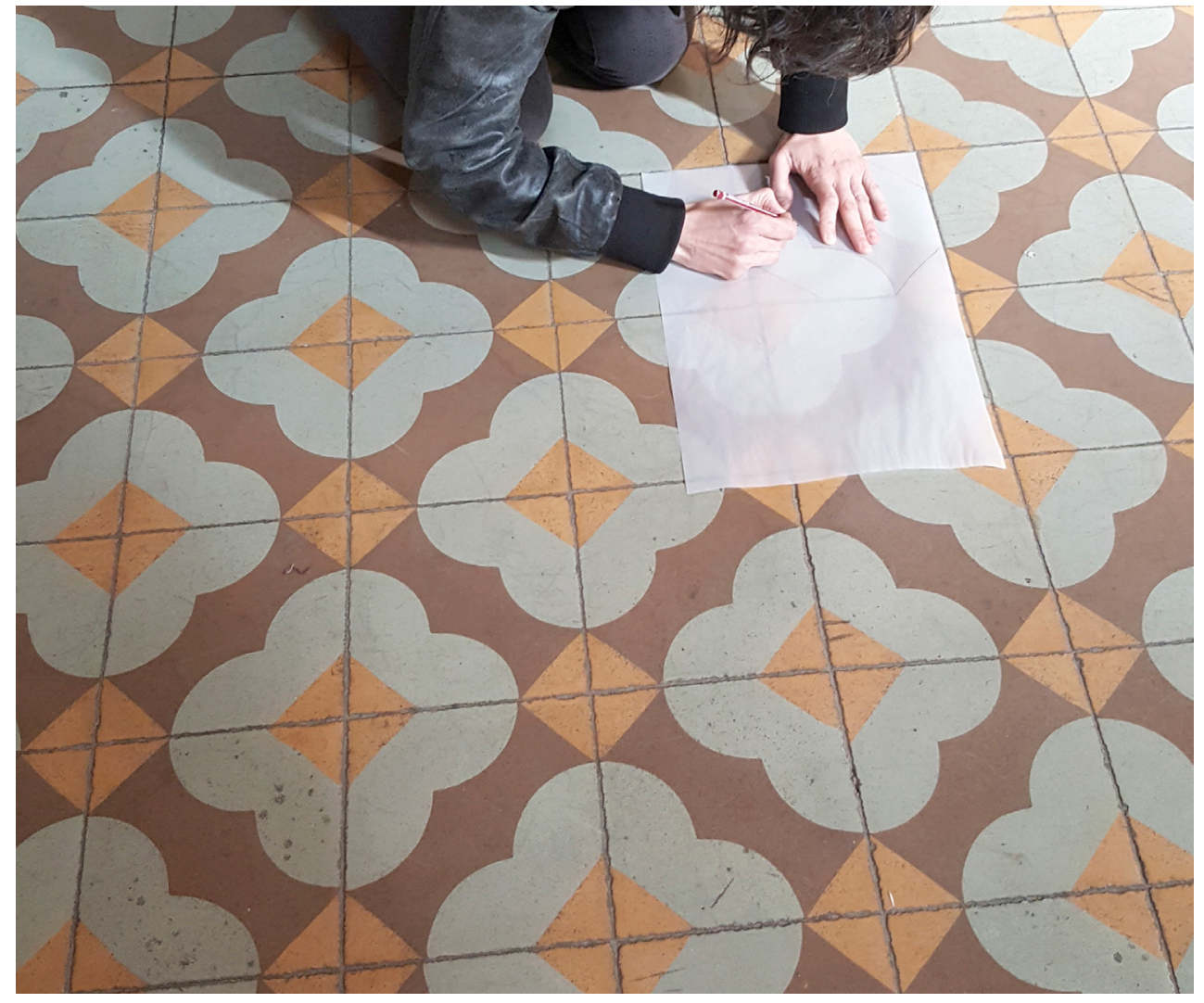

Fig. 3. Rosario Velasco en el proceso de calco del dibujo original del mosaico en la capilla. Fotografía Ángel García Roldán 


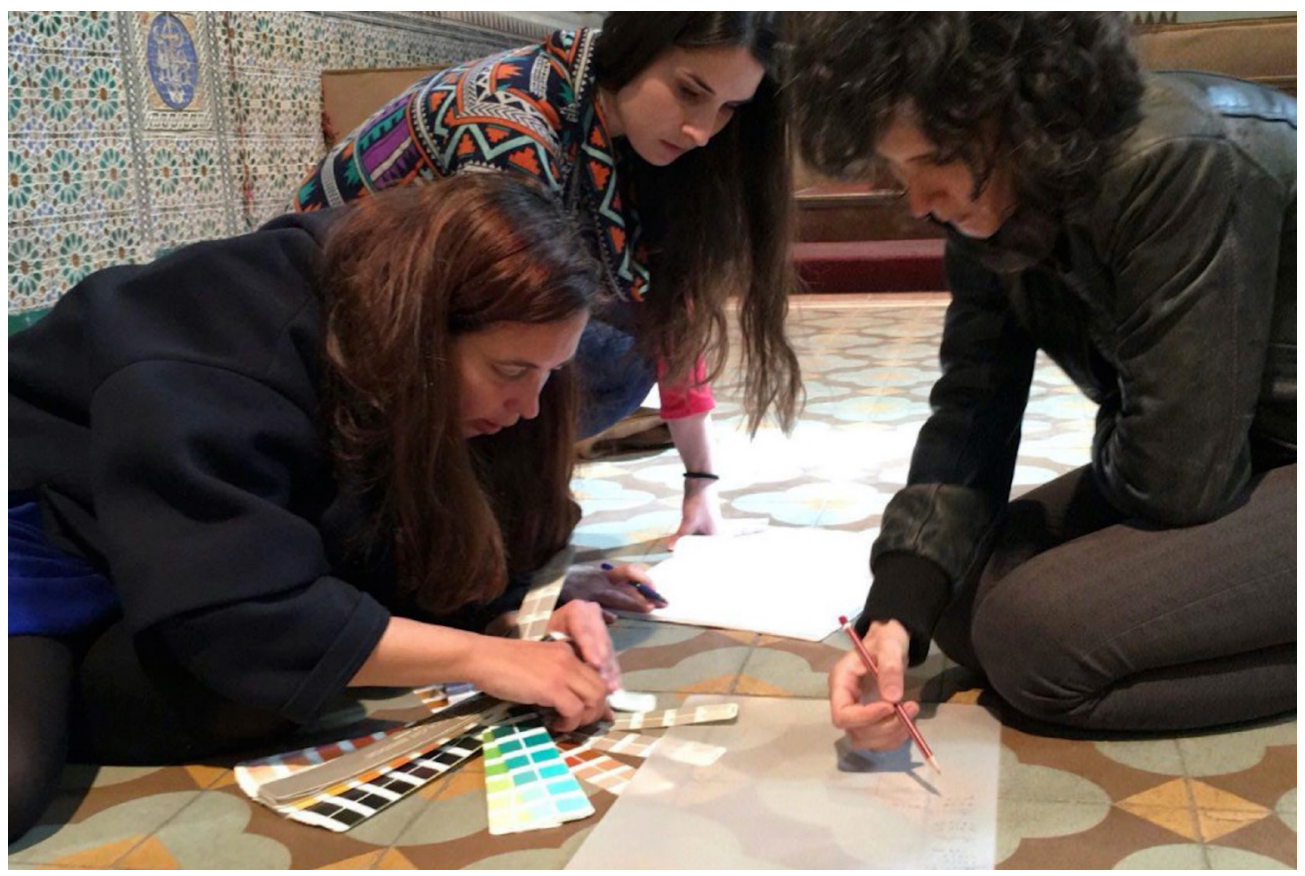

Fig. 4. Rosario Velasco en la elección de color. Fotografía Ángel García Roldán

La idea fue generar un objeto identificativo del patrimonio de la Universidad de Granada, fácil de adquirir, accesible económicamente y sencillo de exponer en cualquier localización. Se realizaron dos versiones: una versión manteniendo el diseño original de la baldosa, con el que puede reproducirse el patrón de este mosaico en amplias superficies como revestimiento constructivo; otra versión especifica como pieza exenta, en la que el motivo original ha sido escalado encajando el mosaico en una única baldosa de $20 \times 20 \mathrm{~cm}$. que, enmarcada en madera de roble, embalada en una caja corporativa con un tarjetón explicativo, se convierte en objeto decorativo singular, potencial regalo o souvenir que actúa como agente transmisor del valor simbólico e iconográfico del patrimonio histórico de la institución.

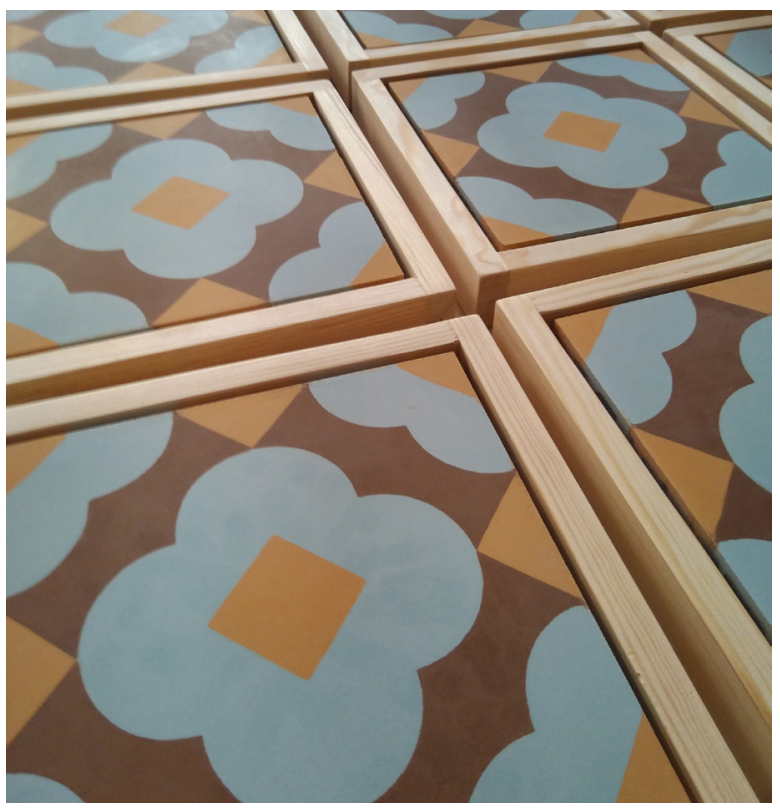

Fig. 5. Baldosa enmarcada. 
Con estas actuaciones La Tienda Universidad de Granada revaloriza la artesanía local. Este novedoso proyecto rescata, impulsa y reconoce procesos de fabricación tradicionales y aporta visibilidad a diseñadores y artesanos emprendedores y comprometidos con el espacio cultural autóctono. El programa ha contribuido a que esta y otras producciones artesanales accedan al público, vinculando la universidad, a través de su tienda, el patrimonio institucional, el tejido productivo y la sociedad. El diseño es aquí un actor destacado, aporta un valor diferencial que orienta el producto artesano hacia un contexto de difusión cultural con interés comercial.
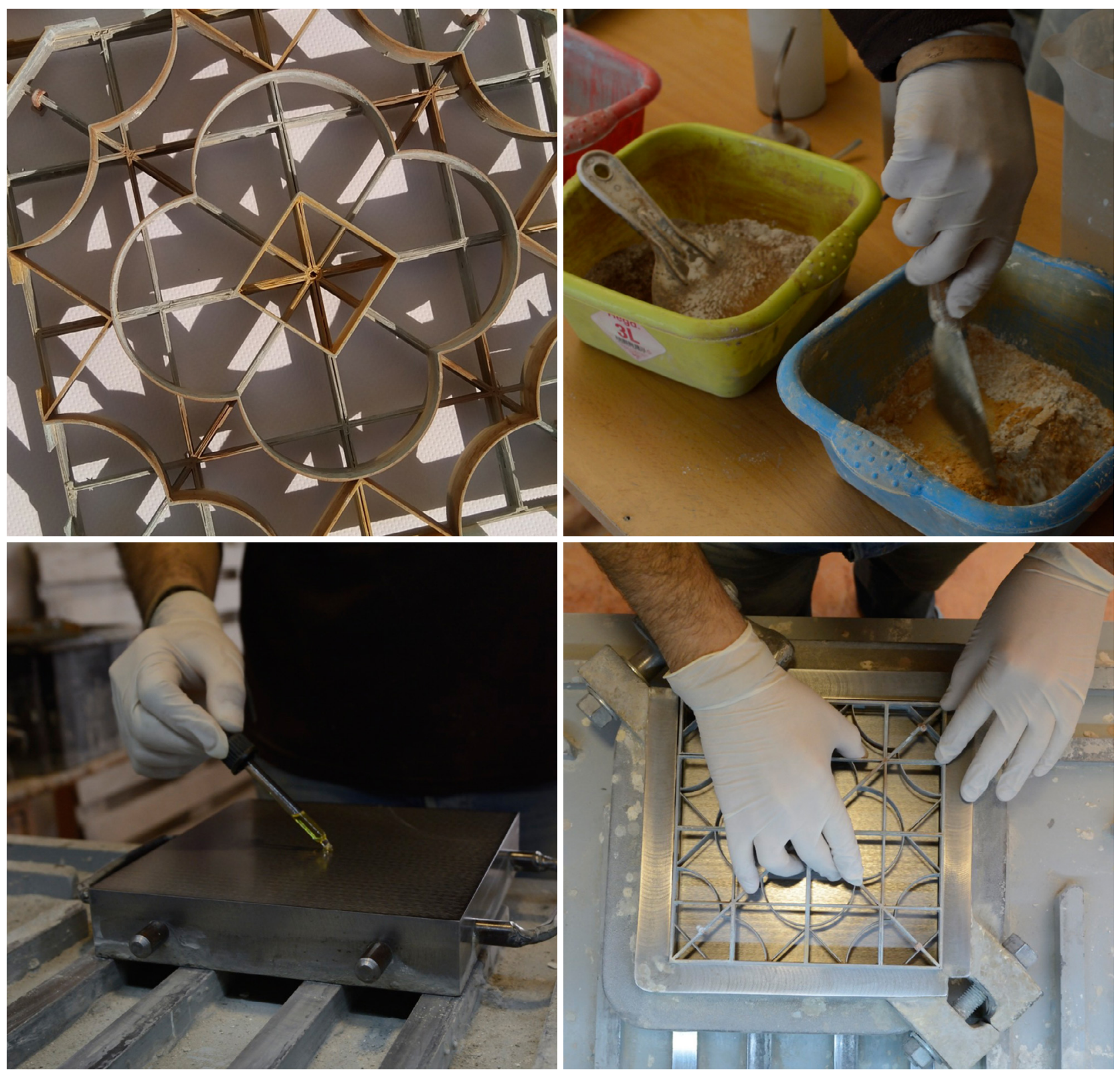

Figs. 6-9. Proceso de creación artesana y manual de la baldosa hidráulica con molde o trepa. Fotografía Juan Linares Aguilar. 

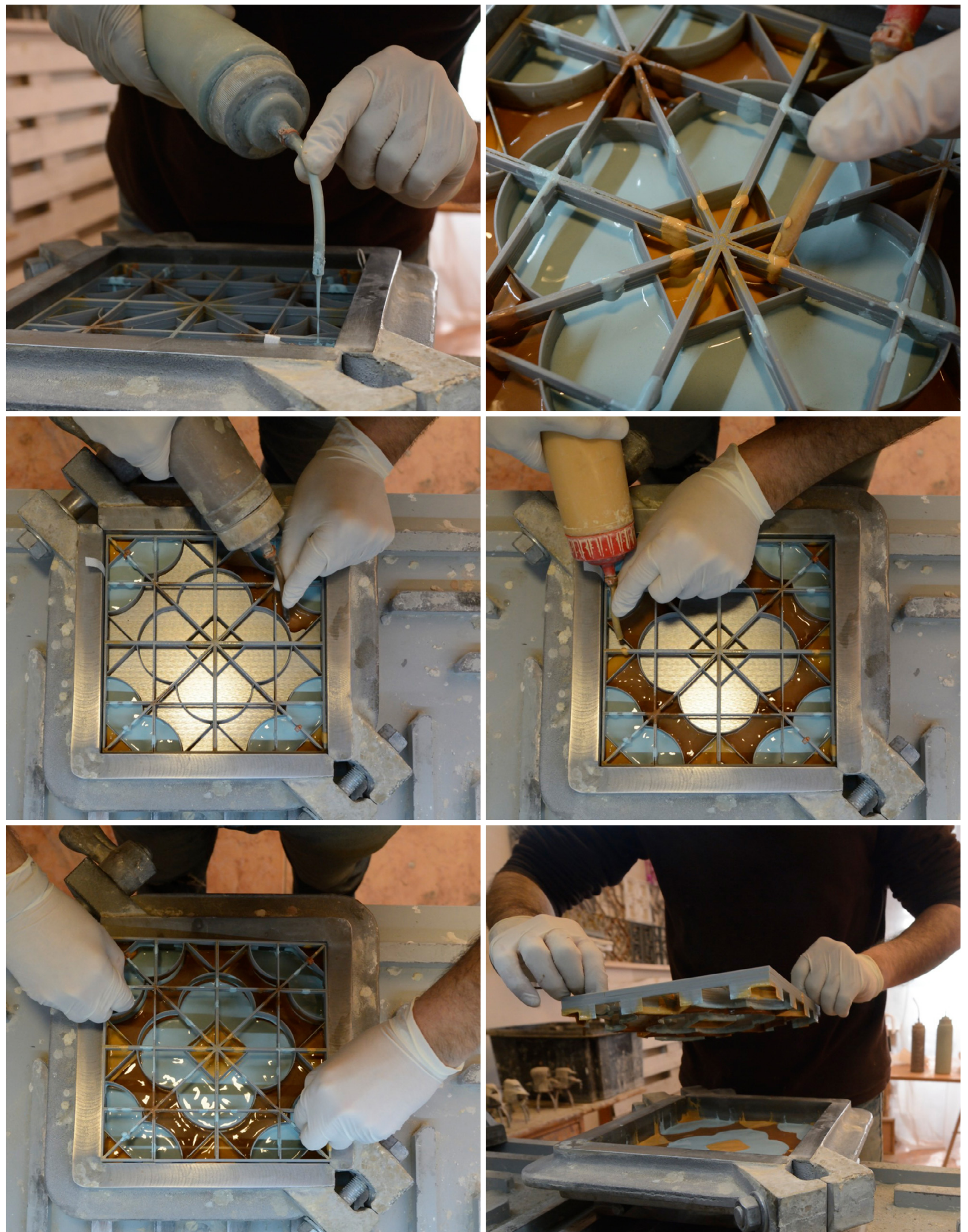

Figs. 10-15. Proceso de creación artesana y manual de la baldosa hidráulica con molde o trepa. Fotografía Juan Linares Aguilar. 
i+Diseño | Artesanado para la comunicación de la identidad cultural y patrimonio. Proyecto réplica de baldosas en la Universidad de Granada

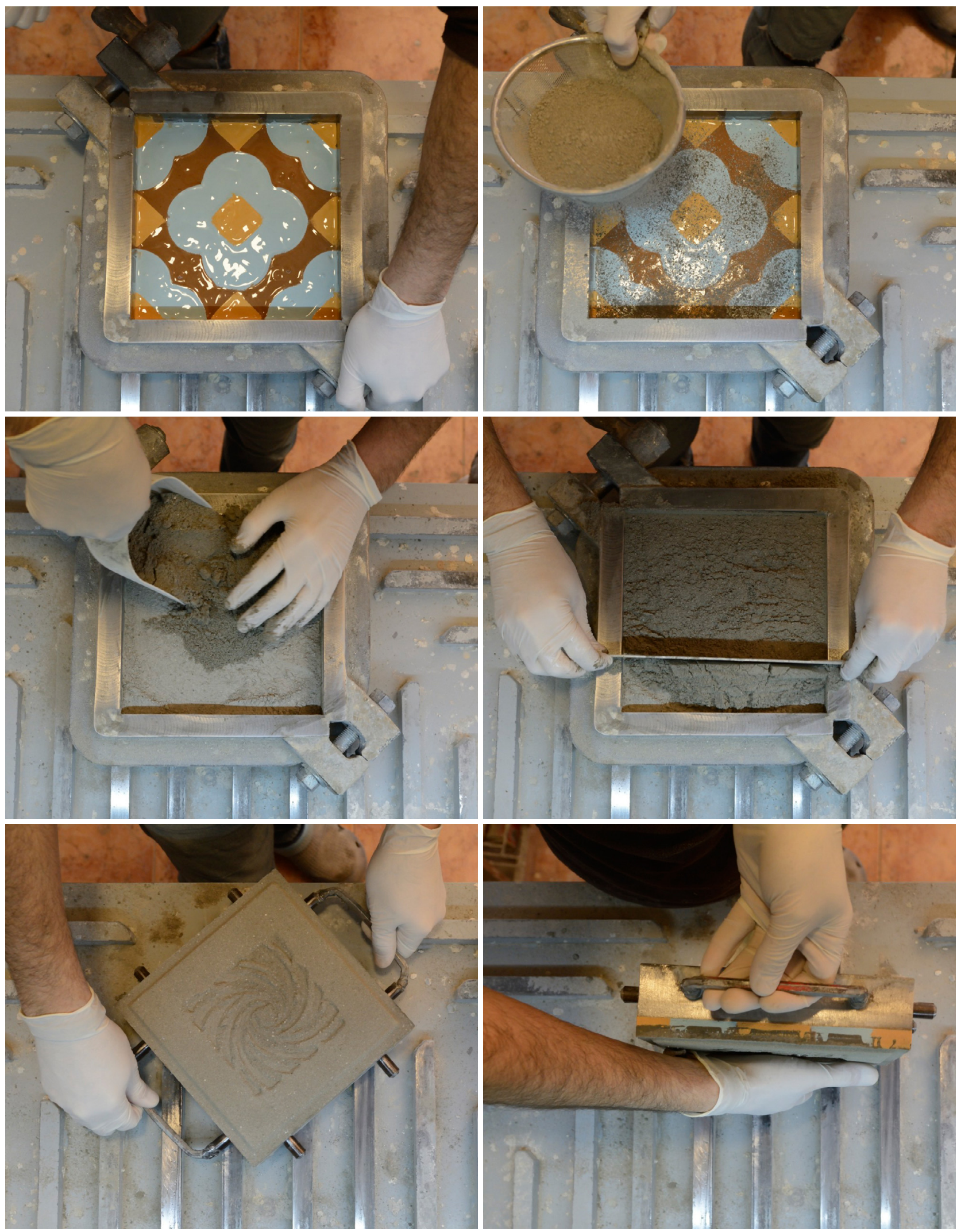

Figs. 16-21. Proceso de creación artesana y manual de la baldosa hidráulica con molde o trepa. Fotografía Juan Linares Aguilar. 


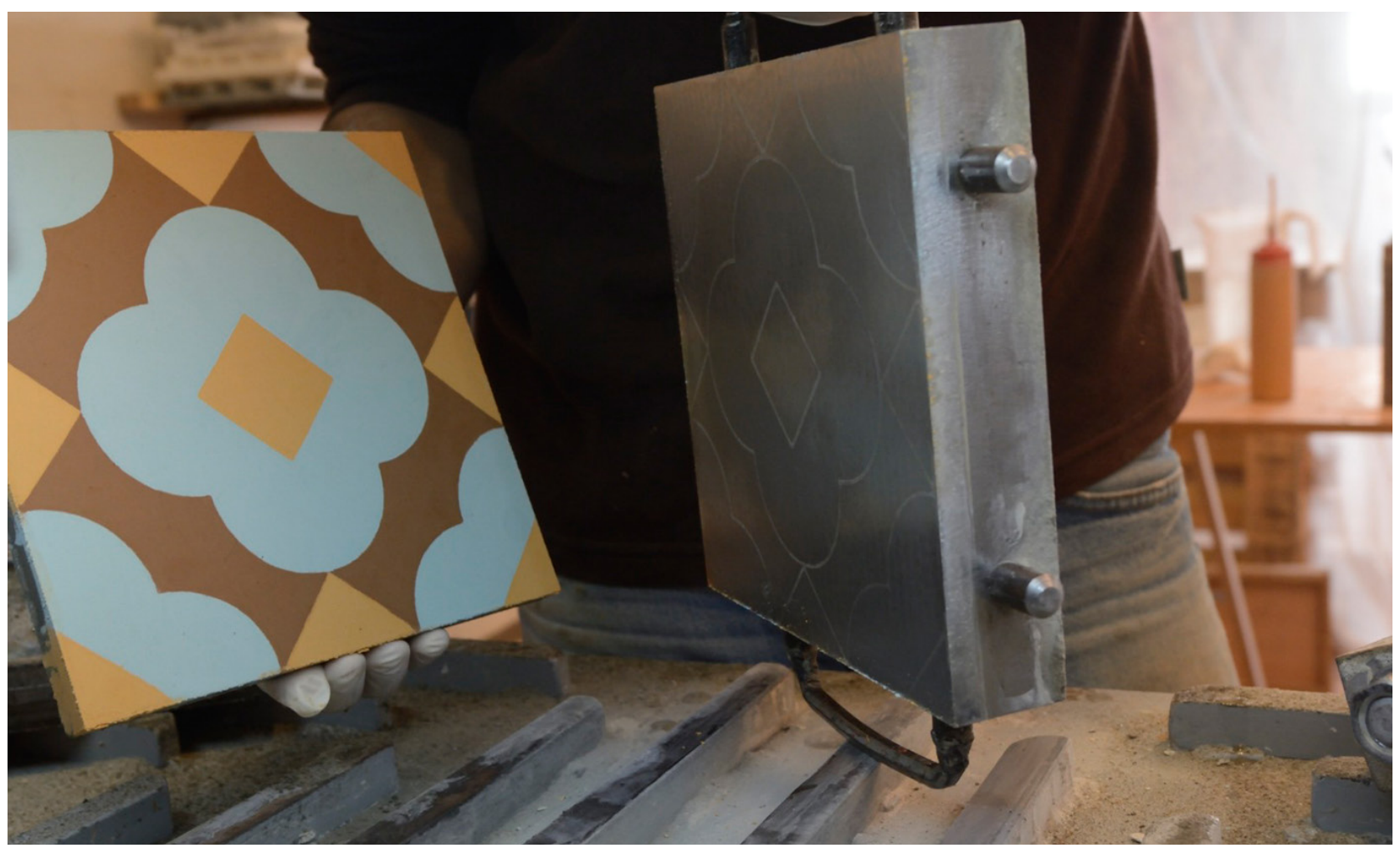

Fig. 22. Proceso de creación artesana y manual de la baldosa hidráulica con molde o trepa.

Fotografía Juan Linares Aguilar.

La Tienda de la Universidad de Granada desarrolla líneas de productos fruto de la unión entre proyectos emprendidos por alumnos egresados —alumnis_, en conjunción e inspiración con el fondo patrimonial de la Universidad de Granada.

Este patrimonio atesora grandes obras y monumentos, vestigios del pasado y huellas del tiempo. Además de su obvia e incalculable aportación a la historia nos regala la posibilidad de su recreación artística. Estas producciones, realizadas apasionadamente, con atención, delicadeza y cuidado, generan objetos de gran belleza, reivindican su vigencia como productos atractivos e interesantes que conectan a la sociedad actual con su cultura.

El Colegio Máximo de Cartuja alberga hoy las facultades de Odontología, de Comunicación y Documentación de la Universidad de Granada. Se trata de una obra realizada por los jesuitas, la primera en Cartuja, entre 1891 y 1894, construida por el arquitecto F. Rabanal. Un monumento histórico-artístico que ha sido declarado Bien de Interés Cultural. Su capilla neomudéjar destaca por unos impresionantes artesonados, y por el excepcional arte musulmán granadino de yeserías con motivos geométricos y vegetales. La nave, de grandes dimensiones — $450 \mathrm{~m} 2$ - , tiene un suelo que está enteramente realizado en baldosa hidráulica. Un bello y agradable diseño geométrico y vegetal propio de los años dorados de la baldosa modernista de finales del siglo XIX, un mosaico con una paleta de colores ocre, tierra y turquesa, que envuelve y aúna el espacio confiriendo solemnidad y elegancia al tiempo que la calidez y ambientación. 


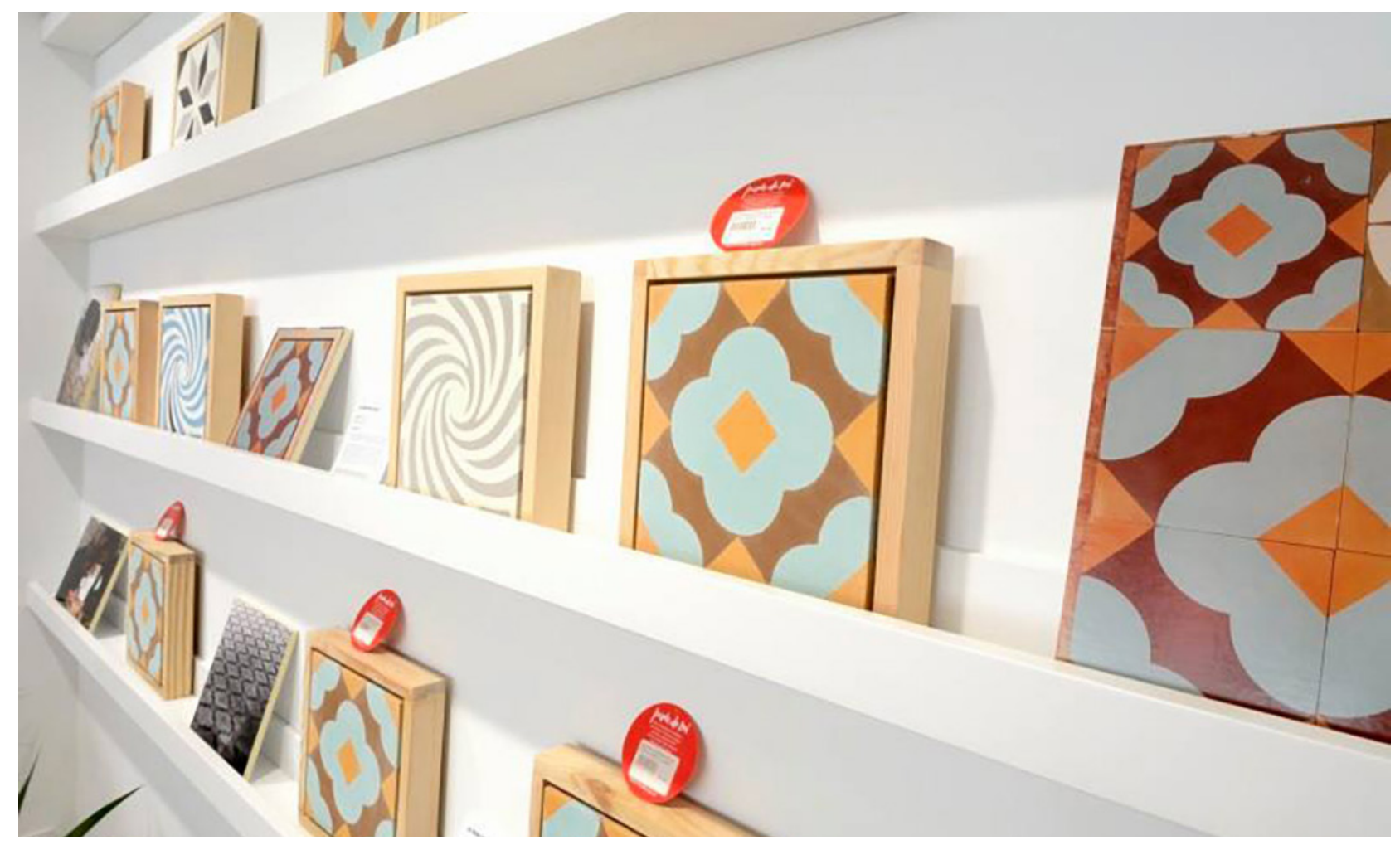

Fig. 23. Exposición en la tienda.

La Universidad de Granada comunica este proyecto dentro de la propia institución, en la ciudad y hacia el exterior reforzando su imagen corporativa. Se configura como un estupendo regalo institucional, que ha dado lugar al desarrollo de una colección derivada que implica a otros artesanos y productos, como pañuelos, abanicos y diversos objetos de merchandising...
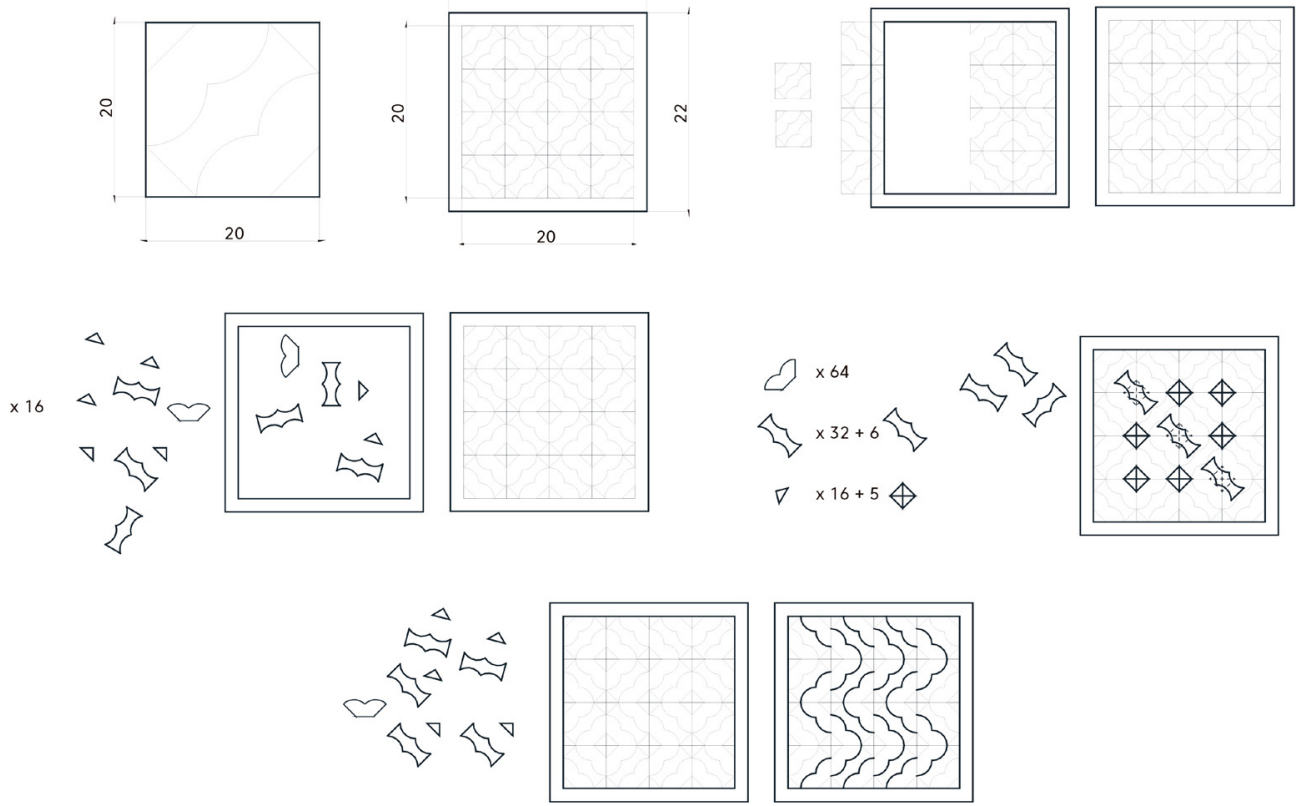

Fig. 24. Puzzle y piezas de madera de pino con barnizado natural. 
i+Diseño | Artesanado para la comunicación de la identidad cultural y patrimonio.

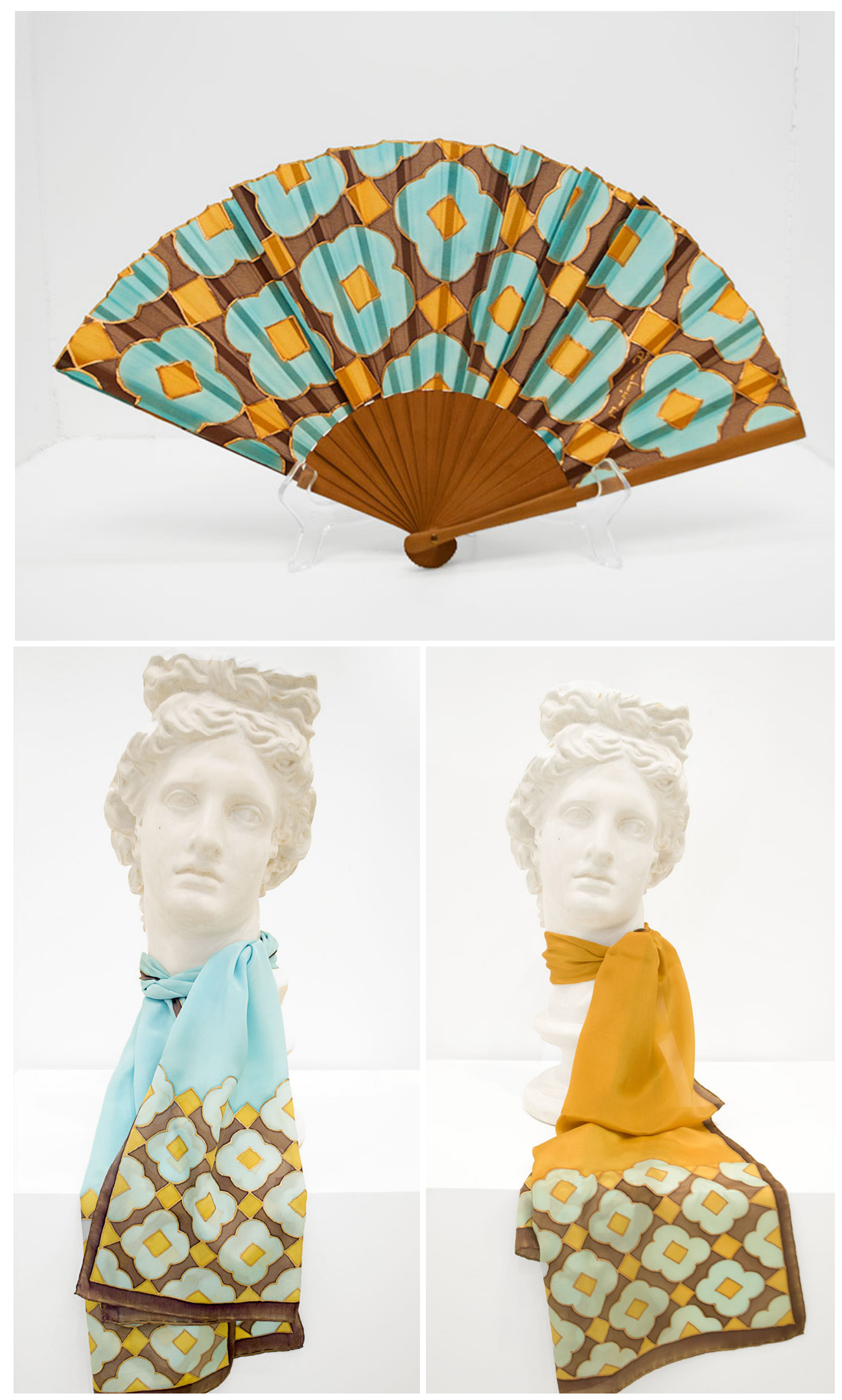

Figs. 25-27. Desarrollo de productos vinculados a la colección, desarrolla- dos por artesanas locales, Pintura en Seda, Mariajoser S.L.U, pintados en seda natural con tintes naturales y fijados al vapor todo a mano. Pañuelos y abanicos. 
Otro punto a tener en cuenta es que la artesanía posibilita la creación y ensayo de prototipos y el encargo de pequeñas producciones, al contrario la producción en serie requiere el pedido de grandes cantidades que tienen en coste excesivo y no permiten el testeo de la muestra. Así se consigue ofrecer un producto exclusivo, de gran calidad, lleno de interés y carácter. Es el fruto del mimo en cada parte del proceso y el manteniendo la técnica original de hace más de un siglo, lo que hace de esta propuesta un proyecto y un objeto de gran singularidad.

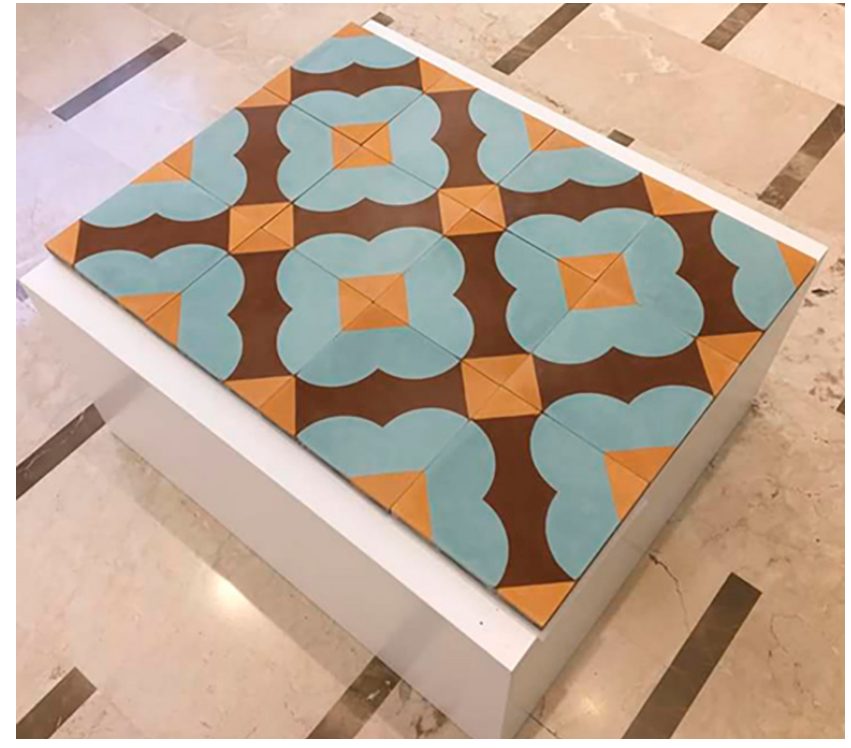

Figs. 28. Reproducción tamaño mosaico original.

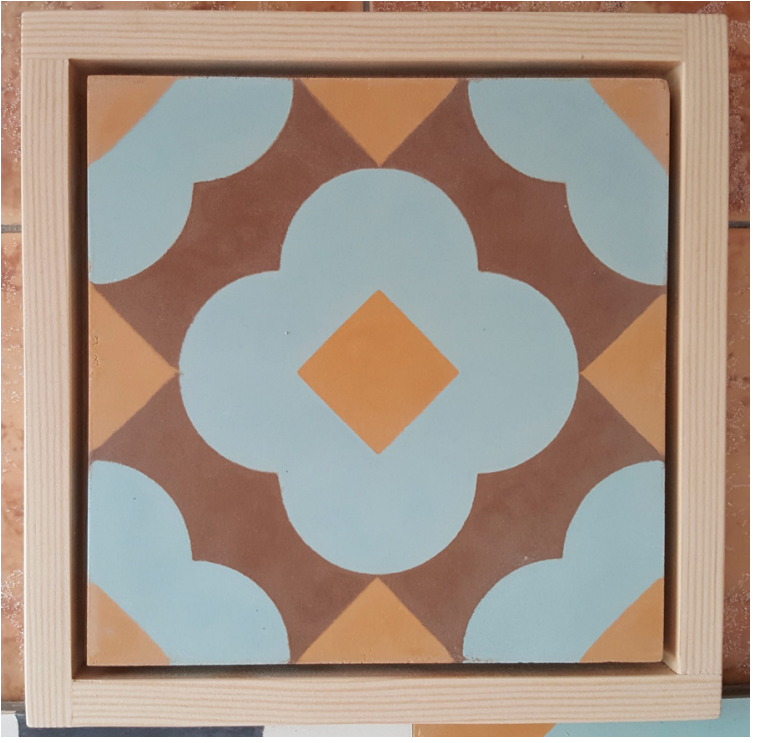

Figs. 29. Baldosa enmarcada.

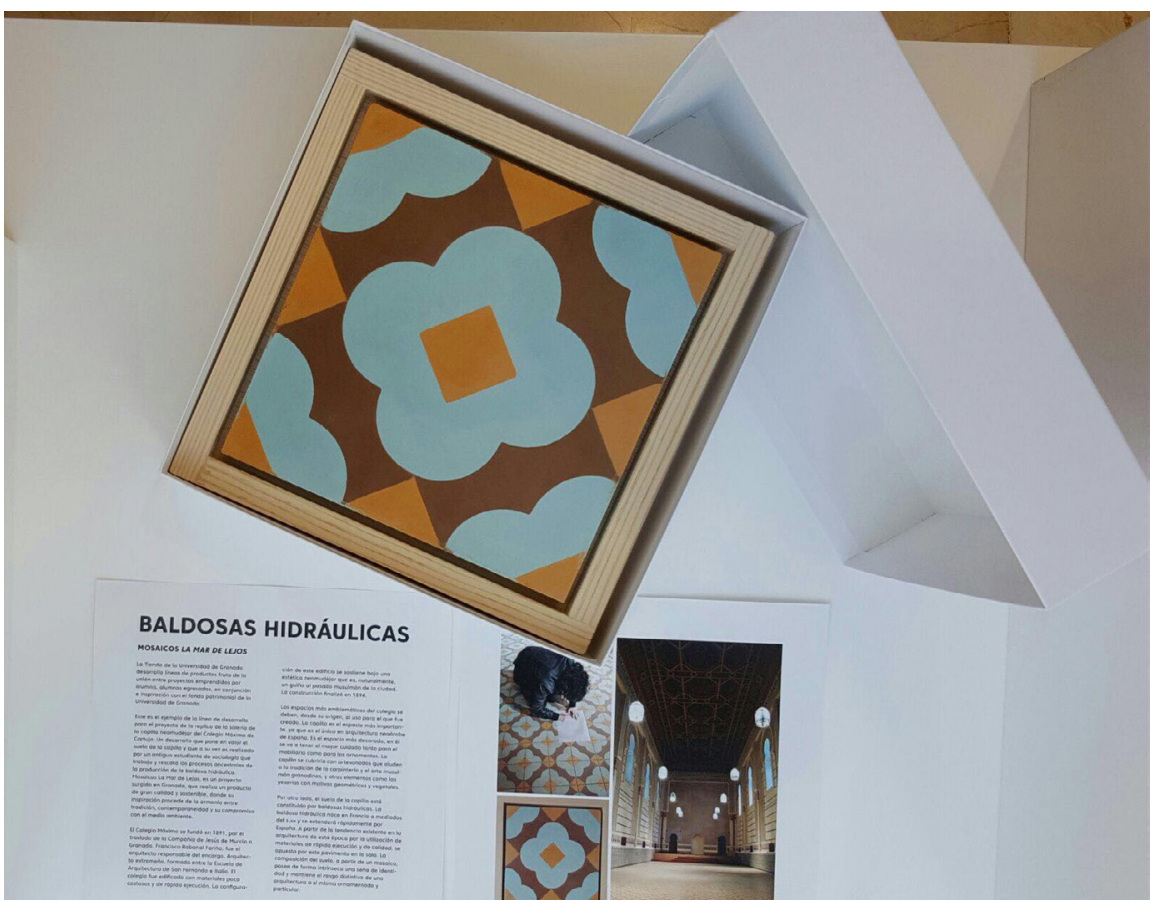

Fig.30. Baldosa enmarcada con tarjetón explicativo. El desarrollo se produjo primero con un proceso, por parte de Rosario Velasco, de calco del dibujo del mosaico original y elección de color de la solería. Posteriormente fue desarrollado por Mosaicos La Mar de Lejos. Así como el packaging que lo alberga, una caja serigrafiada y con el interior forrado, la baldosa envuelta en papel de arroz que la protege, y un tarjetón explicativo donde especifica tanto como ha sido producida, como lo que representa. 
Se precisa, para potenciar su visibilidad y conocimiento, de ciertas estrategias que fomenten la afección hacia el producto y su historia. Así vídeos documentales, talleres, diseño de packaging, storytelling, etc. nos sirven para reforzar el producto y su capacidad de relacionarse con un público consumidor. El 27 de mayo de 2017 se realizó en la tienda un exitoso taller de creación de baldosas hidráulicas, en el que los participantes tuvieron la oportunidad de elaborar un mosaico mediante una experiencia práctica basada en el proceso original de fabricación. Todo ello para acercar al público los sistemas tradicionales de elaboración y producción de este objeto, así como para difundir el artesanado local y el mismo producto.

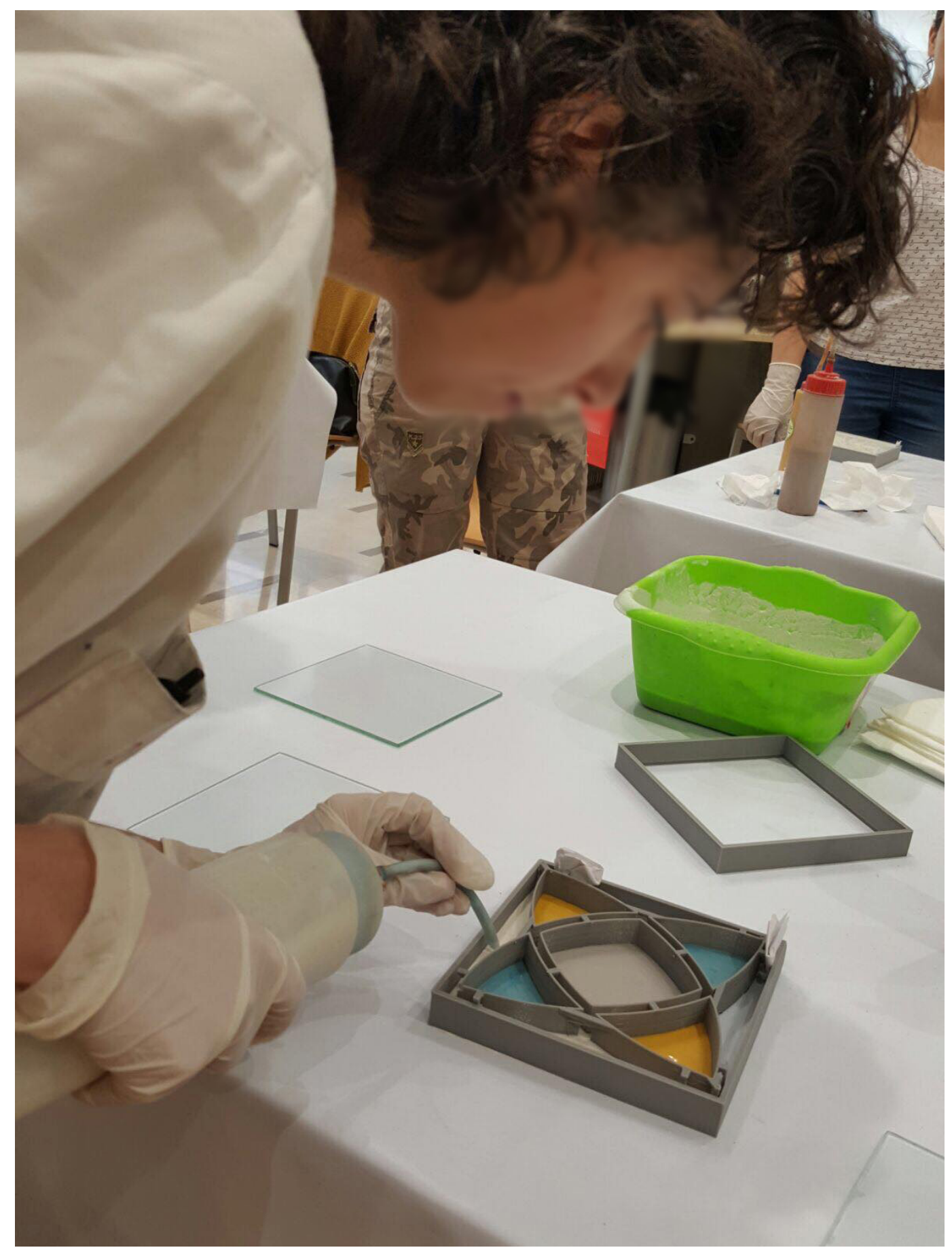

Fig.31. Talleres desarrollados en La Tienda de la Universidad de Granada. 
La nueva Tienda de la Universidad de Granada canaliza y ofrece creaciones dirigidas al comercio promocional de la institución universitaria, se obtiene un posicionamiento de la misma como referente cultural, social y económico de la ciudad, y se demuestran las posibilidades de generar una red de autores y productos con valor añadido y gran idiosincracia. Con estos objetos se crea y genera una propuesta de valor única, concreta y diferente, que la desmarca de cualquier modelo de negocio existente en la ciudad y en otras universidades, reforzando los valores patrimoniales propios de esta institución.

\section{Bibliografía:}

Calvera, Anna (ed). De lo bello de las cosas: materiales para una estética del diseño. Gustavo Gili. Barcelona, 2007.

Calvera, Anna (Editora) VVAA. Arte ¿? Diseño. nuevos capítulos para una polémica que viene de lejos. Gustavo Gili. Barcelona, 2003.

Campi, Isabel. Diseño e historia: tiempo, lugar y discurso. Designio. México, 2010.

Castellone, Eulalia. Artesanías vegetales. Editora Nacional. Madrid, 1982.

Gombrich, Ernst. H. El sentido del orden. Estudio sobre la psicología de las artes decorativas. Gustavo Gili. Barcelona, 1980.

Mora, Tachy. Artesanía española de vanguardia. Innovación y diseño en las industrias artesanales contemporáneas. Lunwerg. Madrid, 2011.

Morris, William. Arte y Artesanía. Cuadernos de Langre. San Lorenzo del Escorial, 2011.

Munari, Bruno. ¿Cómo nacen los objetos? Apuntes para una metodología proyectual. Gustavo Gili. Barcelona, 2010.

Norman, Donald A. El diseño emocional: por qué nos gustan (o no) los objetos cotidianos. Paidós. Barcelona, 2005.

Norman, Donald A. La psicología de los objetos cotidianos. Nerea, Madrid, 1990.

O'Bannon, George. Alfombras orientales. Guía del coleccionista para seleccionar, identificar y disfrutar de la alfombras orientales nuevas y antiguas. Edimat, Madrid, 1999.

Owen, Jones. El patio de la Alhambra en el Crystal Palace. Erigido y descrito por Owen Jones. Abada editores. Madrid, 2010.

Thackara, John. Cómo prosperar en la economía sostenible. Diseñar hoy el mundo de mañana. Experimenta Theoria, Madrid, 2016.

Vvaa. Presente y futuro de las artesanías en la era industrial. Ministerio de Industria y Energía. Madrid, 1982.

\section{Enlaces}

Ley 15/2005, de 22 de diciembre, de Artesanía de Andalucía.

https://www.boe.es/buscar/pdf/2006/BOE-A-2006-759-consolidado.pdf

Estatuto de Autonomía de La Junta de Andalucía

http://www.juntadeandalucia.es/export/drupaljda/lo_2-2007.pdf

Constitución Española

http://noticias.juridicas.com/base_datos/Admin/constitucion.t7.html

Calvera, Anna. «La modernidad de William Morris». Temes de disseny, núm. 14. Elisava TdD. Barcelona, 1997. En: http://www.raco.cat/index.php/Temes/article/view/29505 (consulta 18 de mayo de 2017). 
Campi, Isabel. «El retorn de l'ornament», Temes de disseny, núm. 2. Elisava TdD. Barcelona, 1998. En: http:// www.raco.cat/index.php/Temes/article/view/29053/39863 (consulta 18 de mayo de 2017).

VVAA «Magic box: craft and the computer». Eye Magazine, 2008. En: http://www.eyemagazine.com/feature/ article/magic-box-craft-and-the-computer (consulta 18 de mayo de 2017).

VVAA «The decriminalisation of ornament». Eye Magazine, 2005. En: http://www.eyemagazine.com/feature/ article/the-decriminalisation-of-ornament-full-text (consulta 18 de mayo de 2017).

Para su difusión y promoción se han utilizado diversos canales de comunicación, físicos y digitales: Taller

https://canal.ugr.es/noticia/taller-baldosas-hidraulicas-todoslos-publicos/

Entrevista

https://patrimonio.ugr.es/multimedia/la-mar-de-lejosentrevista-a-jose-angel-valdes/_https://www.youtube. $\mathrm{com} /$ watch?time_ continue $=9 \& \mathrm{v}=8 \mathrm{Z}$ yfzfE $6 \mathrm{FY} 4$

Producto

https://www.facebook.com/tiendaugr/photos

https://tienda.ugr.es/colecciones/alumni/baldosa-hidraulica/ 